\title{
Reflets
}

Revue d'intervention sociale et communautaire

\section{Réalité, enjeux et défis de la pratique du développement communautaire auprès des communautés francophones en situation minoritaire}

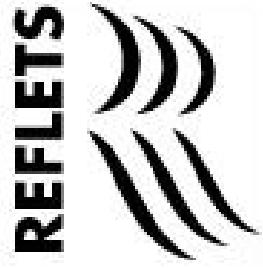

\section{Sébastien Savard et Dominique Charbonneau}

Volume 22, numéro 2, automne 2016

La recherche et l'intervention en travail social en contexte francophone minoritaire au Canada

URI : https://id.erudit.org/iderudit/1038971ar

DOI : https://doi.org/10.7202/1038971ar

Aller au sommaire du numéro

Éditeur(s)

Reflets, Revue d'intervention sociale et communautaire

ISSN

1203-4576 (imprimé)

1712-8498 (numérique)

Découvrir la revue

Citer cet article

Savard, S. \& Charbonneau, D. (2016). Réalité, enjeux et défis de la pratique du développement communautaire auprès des communautés francophones en situation minoritaire. Reflets, 22(2), 69-94. https://doi.org/10.7202/1038971ar
Résumé de l'article

Cet article présente les résultats d'une recherche réalisée par l'entremise d'entrevues semidirigées auprès de neuf intervenantes et intervenants et gestionnaires impliqués dans la réalisation de projets de développement communautaire interpellant les communautés francophones en contexte minoritaire de la région de Champlain, en Ontario. Les résultats, interprétés à la lumière du modèle du capital social, démontrent que les communautés francophones vivent des enjeux qui leur sont propres, tels que l'accès au marché de l'emploi et aux services communautaires en français ainsi que l'adaptation des organismes sociaux et de santé francophones aux nouvelles réalités de la francophonie. Ces enjeux influencent directement la pratique des agentes et agents de développement communautaire qui travaillent avec cette communauté.
Tous droits réservés (C) Reflets, Revue d’intervention sociale et communautaire, 2017
Ce document est protégé par la loi sur le droit d'auteur. L'utilisation des services d'Érudit (y compris la reproduction) est assujettie à sa politique d'utilisation que vous pouvez consulter en ligne. 


\title{
Réalité, enjeux et défis de la pratique du développement communautaire auprès des communautés francophones en situation minoritaire
}

\author{
Sébastien Savard, Ph.D. \\ École de service social, Université d'Ottawa \\ Dominique Charbonneau \\ Étudiante au baccalauréat en service social, Université d'Ottawa
}

\section{Résumé}

Cet article présente les résultats d'une recherche réalisée par l'entremise d'entrevues semidirigées auprès de neuf intervenantes et intervenants et gestionnaires impliqués dans la réalisation de projets de développement communautaire interpellant les communautés francophones en contexte minoritaire de la région de Champlain, en Ontario. Les résultats, interprétés à la lumière du modèle du capital social, démontrent que les communautés francophones vivent des enjeux qui leur sont propres, tels que l'accès au marché de l'emploi et aux services communautaires en français ainsi que l'adaptation des organismes sociaux et de santé francophones aux nouvelles réalités de la francophonie. Ces enjeux influencent directement la pratique des agentes et agents de développement communautaire qui travaillent avec cette communauté.

Mots clés : intervention collective, communautés francophones, développement communautaire, francophonie minoritaire, capital social, travail social, Ontario, services sociaux, santé, enjeux

\section{Abstract}

This paper presents the results of a research project conducted with nine community development agents involved in the implementation of community projects with francophone minority communities in the Champlain region, Ontario. The results demonstrate that francophone communities have to deal with issues that are specific to their realities, such as access to the labour market and community services in their language 
as well as the adaptation of francophone social and health agencies governance to the new realities of the francophone community. These issues directly affect the practice of community development agents working with francophone communities.

Key words: community development, francophones, social capital, linguistic minority community, social work, issues

\section{Introduction}

Peu d'écrits ont été réalisés sur la pratique du développement des communautés en milieu francophone minoritaire. L'état de santé des populations francophones minoritaires et les enjeux entourant la prestation de services de santé à ces communautés ont fait l'objet d'un certain nombre d'écrits scientifiques (Bouchard et Desmeules, 2011; Drolet, et collab., 2014; Groupe de travail sur les services de santé en français, 2005; Santé Canada, 2007), ainsi que les politiques publiques qui les concernent (Cardinal, Lang et Sauvé, 2008; Forgues, Thibault et Cardinal, 2010; Forgues, Allard et Landry, 2012; Landry, Forgues et Traisnel, 2010). La littérature francophone sur la pratique professionnelle du développement communautaire a surtout documenté l'organisation communautaire telle que pratiquée au Québec, grâce, entre autres, à de nombreuses monographies publiées depuis 30 ans sur le sujet (Lamoureux, Mayer et Panet-Raymond, 1984;

"... dans chacun des centres de ressources communautaires ayant pignon sur rue dans des communautés à forte présence francophone, on trouve des professionnelles et professionnels qui travaillent quotidiennement avec des groupes du milieu dans le but de trouver des solutions collectives aux difficultés qu'ils rencontrent. »
Doucet et Favreau, 1993; Favreau et Hurtubise, 1993; Lamoureux, et collab., 2002; Bourque, et collab., 2007; Bourque et Lachapelle, 2010). De plus, les organisatrices et organisateurs communautaires québécois sont regroupés dans une association provinciale depuis 25 ans, et un cadre de référence a été rédigé par ce regroupement afin de baliser les principes, les valeurs, la mission et les modèles véhiculés par cette profession. Cette formalisation et cet encadrement ont grandement contribué à la reconnaissance de l'organisation communautaire comme profession au Québec.

En Ontario francophone, l'intervention communautaire, comme pratique professionnelle, a peu bénéficié d'un tel soutien de la littérature scientifique pour définir et valoriser son rôle dans le développement des communautés et dans l'amélioration du bien-être des populations les plus démunies. Mais cela ne veut pas dire qu'il ne se fait pas d'intervention 
communautaire en Ontario francophone. Au contraire, dans chacun des centres de ressources communautaires ayant pignon sur rue dans des communautés à forte présence francophone, on trouve des professionnelles et professionnels qui travaillent quotidiennement avec des groupes du milieu dans le but de trouver des solutions collectives aux difficultés qu'ils rencontrent. C'est donc en partie pour contribuer à une meilleure connaissance des réalités de la pratique du développement des communautés en Ontario francophone que nous avons réalisé une recherche exploratoire auprès d'agentes et agents de développement communautaire (ADC) travaillant avec les communautés francophones minoritaires de la région de Champlain, en Ontario.

L'objectif de la recherche qui est à la base de cet article était de mieux documenter les particularités de la pratique de l'intervention communautaire en Ontario francophone. Quels sont les fondements théoriques et méthodologiques sur lesquels les ADC appuient leur pratique? Comment définissent-ils leurs rôles et leurs mandats dans la communauté? Quels sont les valeurs et les principes fondamentaux qu'ils défendent? Quelles relations entretiennent-ils avec les autres intervenantes et intervenants de leur organisation? Quel impact a sur leur pratique le fait d'intervenir auprès d'une communauté d'identité caractérisée par leur appartenance à la minorité linguistique de la francophonie? Voilà le genre de questions auxquelles nous avons cherché à répondre dans le cadre de cette étude.

Nous commençons cet article en présentant le cadre théorique qui encadre l'analyse et l'interprétation des résultats de notre démarche. Nous avons décidé de retenir le modèle du capital social développé par Coleman (1988), Bourdieu (1986) et Putnam (1995) pour discuter des pratiques de développement des communautés cherchant à améliorer les conditions de vie dans les communautés francophones minoritaires de la région de Champlain, en Ontario. Nous croyons que ce cadre permet de mettre en lumière une des fonctions centrales de l'intervention communautaire, soit de favoriser la création et l'intensification des liens entre les membres d'une communauté (bonding) et entre la communauté et les autres ressources environnantes pouvant soutenir l'action dans le milieu local (bridging). Nous exposons ensuite la méthodologie de recherche retenue pour recueillir et analyser les données. Nous continuons avec la présentation des résultats, section qui se divise elle-même en deux parties : la première, où nous dégageons les principales caractéristiques des agentes et agents de développement communautaire qui travaillent en Ontario francophone, et la deuxième, qui s'attarde plus spécifiquement aux enjeux et défis relatifs à la pratique du développement communautaire réalisée avec les communautés francophones en situation minoritaire. Enfin, dans la section «Discussion et conclusion ", nous faisons des liens entre les résultats de la recherche et le cadre d'ana- 
lyse du capital social tel que défini par Ruuskanen. Cette démarche fait ressortir les forces et les faiblesses de la communauté francophone en situation minoritaire pour soutenir le développement de son capital social et le rôle que peuvent jouer les agentes et agents de développement communautaire dans l'atteinte de cet objectif.

\section{Le modèle du capital social}

Le politologue américain Robert Putnam est l'auteur ayant le plus contribué à populariser le concept et le modèle théorique du capital social. Selon Putnam, le capital social peut être défini comme "les caractéristiques d'organisations sociales, telles que les réseaux, les normes et la confiance, qui facilitent la coordination et la coopération en vue de produire un bénéfice mutuel " [notre traduction] (Putnam, 1995, p. 67). Pour Putnam, donc, les communautés qui s'en sortent le mieux sont celles qui favorisent et valorisent l'engagement citoyen et où des normes centrées sur la confiance et la réciprocité se

"Dans les communautés où les gens ont davantage tendance à se regrouper et à s'associer, surtout dans des organismes communautaires ou des groupes bénévoles, les membres participent davantage à la vie démocratique de leur communauté et se mobilisent plus facilement pour faire face aux défis et aux enjeux auxquels est confrontée leur collectivité. » sont développées. Dans les communautés où les gens ont davantage tendance à se regrouper et à s'associer, surtout dans des organismes communautaires ou des groupes bénévoles, les membres participent davantage à la vie démocratique de leur communauté et se mobilisent plus facilement pour faire face aux défis et aux enjeux auxquels est confrontée leur collectivité.

Putnam a identifié deux dimensions autour desquelles se construit le capital social d'une communauté, soit le bonding, qui réfere aux liens et relations qui existent entre les membres d'une même communauté, et le bridging, qui correspond aux relations qu'une communauté entretient avec d'autres communautés ou avec des institutions situées à l'extérieur de la communauté. À l'intérieur des relations de type bridging, on retrouve également les liens entre la communauté et le pouvoir formel, comme le gouvernement local et l'État central. À la lumière de cette catégorisation, une communauté qui jouit d'un capital social élevé est une communauté qui se distingue, d'une part, par des liens forts et dynamiques entre les membres qui y évoluent, ce qui est permis et facilité par la présence de normes sociales solidaires intenses, partagées et solidifiées par un niveau de confiance mutuelle important, et, d'autre part, par des liens multiples, 
diversifiés et productifs avec des communautés et des institutions situées à l'extérieur de la communauté, qui enrichissent le capital social de celle-ci en permettant de tirer profit de ressources absentes de la communauté. Ainsi, selon Bridge, Murtagh et O’Neill :

L'accès à un niveau important de capital social sous la forme d'organisations, de réseaux, de confiance et de normes communes peut constituer un fondement important permettant la mobilisation économique locale et favorisant la création d'un cercle vertueux de croissance économique et de développement social dans une perspective de développement communautaire durable. [notre traduction] (Bridge, Murtagh et O’Neill, 2009, p. 180)

À l'instar de Putnam, d'autres auteurs ont contribué au développement et au renouvellement de modèles théoriques liés au capital social. Ruuskanen (2001), entre autres, propose un modèle qui répond en partie à une question ou à une tension souvent soulevée, surtout à la suite des écrits de Putnam concernant ce qui appartient aux déterminants (ou causes) du capital social ou à ses effets. En d'autres termes, la cohésion sociale, l'implication citoyenne dans les organismes bénévoles et la confiance mutuelle sont-elles des déterminants du capital social, ou sont-elles plutôt des effets ou des résultats de celui-ci? Ruuskanen (2001) suggère d'envisager le capital social comme une notion composée de trois éléments : les déterminants, les mécanismes et les retombées du capital social. Dans sa recension des écrits, Martin-Caron (2013) présente une figure qui reprend

"...Bouchard et collab. (2006) ... font bien ressortir l'importance de sintéresser au capital social de cette communauté comme ressource pouvant soutenir la vitalité de la francophonie et soulignent que cette même vitalité peut en même temps, dans une dynamique circulaire, alimenter ce même capital social." ces dimensions (voir la figure 1 ci-dessous). On comprend donc qu'il existe des sources du capital social qui relèvent des individus, alors que d'autres appartiennent à la communauté visée (méso), de même qu'à la société qui englobe ces deux entités (macro). Par conséquent, les individus, les communautés et les sociétés peuvent présenter des intensités variables de certaines composantes faisant fluctuer le niveau de capital social présent chez un individu ou dans une communauté. Les composantes sont différentes lorsqu'elles sont associées à des sources individuelles, communautaires ou sociétales. Selon ce même modèle, certains mécanismes vont contribuer au développement du capital social, autant des individus que des communautés.

Il est à noter que Bouchard, et collab. (2006) ont déjà utilisé le cadre du capital social pour étudier la situation de 
Figure 1 - L'articulation des dimensions du capital social selon Ruuskanen

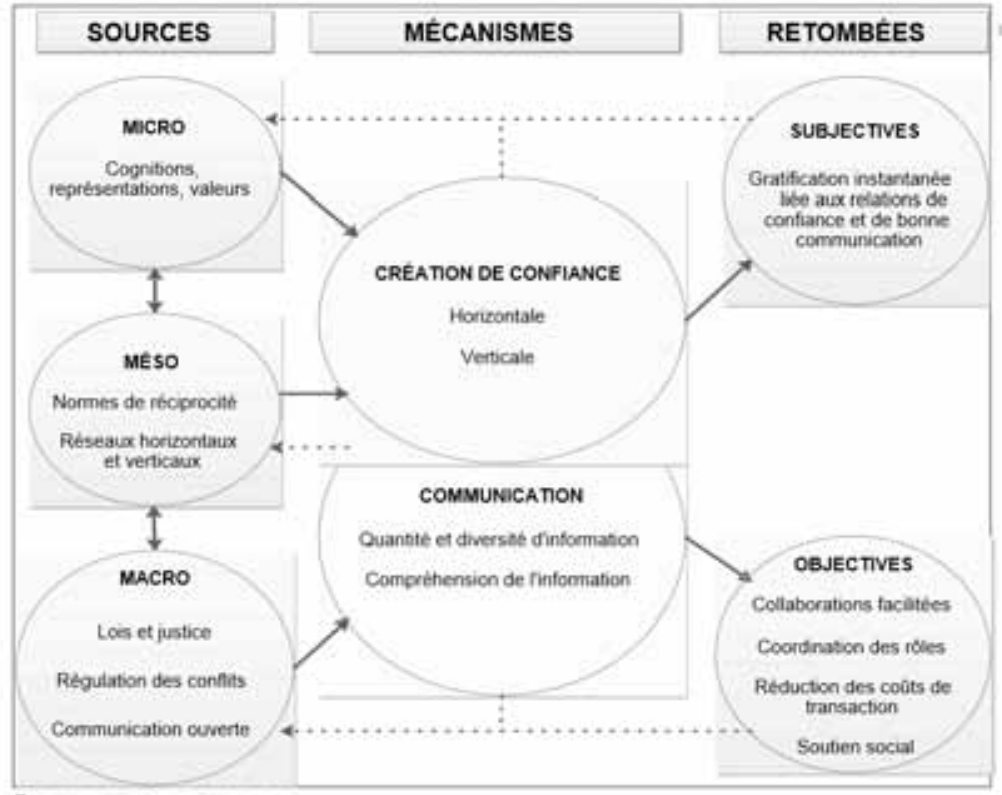

Source : Martin-caren, 2013, p, 15

"...le capital social développé grâce à ces mécanismes va générer des retombées importantes pour les individus et les communautés en termes d'accès à des ressources et à des occasions de croissance, mais également en matière de création de climats favorables à la collaboration et au soutien mutuel." la francophonie minoritaire au Canada. Dans leur article, ces auteures et auteurs font bien ressortir l'importance de s'intéresser au capital social de cette communauté comme ressource pouvant soutenir la vitalité de la francophonie et soulignent que cette même vitalité peut en même temps, dans une dynamique circulaire, alimenter ce même capital social. Dans le présent article, nous voulons démontrer comment les agentes et agents de développement communautaire peuvent occuper une position centrale dans le développement et l'enrichissement du capital social de la communauté francophone en agissant à un niveau local et communautaire.

Ces mécanismes permettent de créer et d'entretenir la confiance entre les membres de la communauté, ainsi qu'entre la communauté et les autres communautés, et ils favorisent également le développement de canaux de 
communication permettant d'accéder à de l'information de qualité sur les enjeux et les possibilités qui se présentent aux individus et aux communautés. De plus, le capital social développé grâce à ces mécanismes va générer des retombées importantes pour les individus et les communautés en termes d'accès à des ressources et à des occasions de croissance, mais également en matière de création de climats favorables à la collaboration et au soutien mutuel.

\section{Méthodologie}

La méthodologie de recherche retenue a reposé sur deux stratégies de collecte de données, soit l'analyse documentaire et la réalisation d'entrevues semi-dirigées. L'analyse documentaire a porté sur l'ensemble de la documentation produite par les organismes qui embauchent des ADC pour définir la nature de leur poste, les tâches associées à leur fonction de même que leurs conditions de travail.

Les entrevues semi-dirigées ont été réalisées avec les intervenantes et intervenants communautaires qui travaillent dans des centres de ressources communautaires, dans des centres de santé et de ressources communautaires et dans un organisme communautaire dont la mission est de soutenir la mise sur pied de ressources répondant à des besoins de la population locale. Ces professionnelles et professionnels, pour être retenus, devaient avoir pour mandat d'intervenir auprès des populations francophones dans la région d'Ottawa et de Prescott-Russell.

Les entrevues abordaient des thématiques telles que les fonctions des intervenantes et intervenants communautaires, les valeurs qu'ils défendent, les fondements et les principes de base de leur intervention, leur formation, leur vision de leur rôle par rapport à celui de la communauté, et les modèles de pratiques qu'ils adoptent. La spécificité du travail avec des communautés francophones minoritaires était également au centre de la discussion.

La codification et le traitement des informations obtenues lors des entrevues ont été réalisés avec le logiciel Nvivo, version 8 , et l'analyse visait à faire ressortir les convergences entre les organisatrices et organisateurs communautaires, de même que les facteurs pouvant expliquer certaines différences dans les expériences des organisatrices et organisateurs communautaires. 


\section{Présentation des résultats}

\section{Présentation des personnes interviewées et de leur cheminement professionnel et personnel}

Au total, cinq agentes et agents de développement communautaire, deux promotrices de la santé, un agent de santé communautaire, ainsi qu'une directrice d'un organisme communautaire ayant pour mandat de soutenir la mise en place d'initiatives répondant à de nouveaux besoins dans la communauté, ont été interviewés. Les entretiens ont porté sur diverses thématiques, dont leur cheminement personnel et professionnel, les fondements théoriques et méthodologiques sur lesquels ils appuient leur pratique, leurs rôles et leurs mandats dans la communauté, sans oublier le contexte organisationnel dans lequel ils travaillent et la particularité de leur intervention en Ontario français.

\section{Formation académique et fondements théoriques}

Nous avons noté des différences marquées entre les cheminements professionnels et personnels des intervenantes et intervenants ayant tracé la voie vers des fonctions

"Le développement des communautés n'apparaît donc pas comme une pratique qui attire les personnes diplômées en début de carrière. C'est davantage un intérêt qui se développe lorsque les intervenantes et intervenants sont en contact dans leur milieu avec des professionnelles et professionnels qui font ce travail, ou parce qu'une opportunité se présente. » d'intervention en organisation communautaire. En effet, leur formation académique est très diversifiée. Le service social est la seule discipline qui se trouve dans le curriculum de plusieurs (soit deux au niveau universitaire et un au niveau collégial). Les autres ont eu une formation dans différentes disciplines des sciences sociales (psychologie, sociologie, études des femmes), ou en éducation et en sciences de l'éducation physique. Tous les intervenants et intervenantes en développement communautaire détiennent minimalement un baccalauréat, tandis que les promotrices de la santé et l'agent de santé communautaire détiennent un baccalauréat ou une formation collégiale. Un fait intéressant, tout de même, c'est que le parcours académique de la majorité est caractérisé par le passage d'une intervention axée sur l'individu à une intervention axée sur le communautaire. Le développement des communautés n'apparaît donc pas comme une pratique qui attire les personnes diplômées en début de carrière. C'est davantage un intérêt qui se développe lorsque les intervenantes et intervenants sont en contact dans 
leur milieu avec des professionnelles et professionnels qui font ce travail, ou parce qu'une opportunité se présente.

"J'ai commencé comme responsable des bénévoles au centre. Deux mois après, mon directeur m'a dit : "Ah ben, notre agente de développement va en congé de maternité, et je vois que tu es capable de faire la job." C'est vraiment comme ça que je suis rentré ici pour être agent de développement. » (ADC 1)

La grande majorité des répondantes et répondants nous ont dit que leur formation collégiale et universitaire avait contribué à les préparer à leur emploi, mais pas de façon importante. Selon plusieurs, c'est l'expérience sur le terrain qui est la source de formation la plus importante :

«Être agent de développement communautaire, ce n'est pas juste rencontrer les gens; c'est aussi avoir une créativité, être capable de dire OK, voici un besoin dans ma communauté, comment je vais faire pour répondre à ces besoins-là. [...] Faut que tu développes des initiatives. Pour développer des initiatives, je veux bien, mais le côté universitaire ne te donne pas ces outils-là. » (ADC 3)

"Ce sont les années dexpérience de travail qui servent de formation pour lintervenant. Dans cette carrière-ci, c'est vraiment eh sur le terrain qu'on apprend beaucoup de choses parce que, chaque jour, je fais de nouvelles expériences [...] y a beaucoup de choses qui sont nouvelles pour nous en même temps parce que c'est le sujet à ce moment-là. » (ADC 8)

La faible influence que semble avoir la formation académique sur le développement professionnel s'explique peut-être en partie par la réalité spécifique de cette fonction, mais elle peut également s'expliquer par un autre phénomène, soit l'absence de formation spécifiquement dédiée à la pratique du développement communautaire dans les universités et les collèges. Les programmes de service social comprennent tous des cours en intervention collective, mais ils sont souvent peu nombreux et occupent une place plutôt ténue dans le programme. Cette absence ou quasi-absence de formation rend difficile la pérennité et le transfert des connaissances et des compétences développées et la constitution d'un patrimoine qui nourrit la pratique et qui assure une certaine forme de standardisation des pratiques. Cette lacune au niveau de la formation académique explique peut-être également, en partie, pourquoi les agentes et agents de développement ont été très peu loquaces en ce qui a trait aux fondements théoriques qui les inspirent et les guident dans leurs interventions. Lorsqu'interrogés sur cette question, les répondantes 
et répondants ont surtout mentionné le partage avec les collègues et la stratégie " no community left behind ", adoptée par la Ville d'Ottawa pour favoriser l'implication d'un ensemble d'actrices et acteurs, en plus des citoyennes et citoyens, dans six quartiers défavorisés, autour de la question de la prévention du crime. Cette approche propose une méthode d'intervention en cinq étapes plutôt classiques : constitution d'un comité de pilotage, évaluation des besoins du milieu, élaboration du plan d'action, implantation des projets, évaluation des résultats. Plusieurs des agentes et agents rencontrés ont été impliqués dans l'implantation de cette stratégie dans leur territoire. Sans être dénuée d'intérêt, elle ne propose pas d'assises théoriques pour expliquer l'existence des problèmes sociaux, le rôle des communautés dans leur résolution et les meilleures stratégies pour soutenir ces milieux.

\section{Rôles et mandats}

Lorsque l'on demande aux répondantes et répondants de définir comment ils voient leurs rôles et leurs mandats dans la communauté, on observe qu'ils se perçoivent souvent comme des outils pour la communauté, comme une ressource qui est à la disponibilité du milieu pour le soutenir dans la recherche de solutions aux problèmes auxquels il est confronté. Ce rôle général se décline en différentes fonctions qui reprennent assez bien celles qui ont été définies par Lee (2011) dans son livre Pragmatics of Community Organization.

\section{Initiateur, catalyseur}

Plusieurs agentes et agents de développement considèrent qu'un de leurs rôles est d'initier des projets dans leur communauté afin répondre à un besoin social ou de santé. Ils doivent être la bougie d'allumage, sortir le milieu de l'apathie.

" Je me verrais comme une clé de contact d'une voiture. C'est-à-dire que je prends la communauté comme le moteur et je prends le centre où je travaille comme la voiture elle-même. Moi je suis comme la clé qui démarre, qui déclenche. Je me vois comme un déclencheur dans le sens qu'une fois que je déclenche, le moteur continue de tourner de soi-même. " (ADC 1)

"On s'attend de moi que je sois le catalyseur de nouveaux projets, celui qui fait bouger les choses dans la communauté. » (ADC 8) 


\section{Celui qui encourage, qui soutient}

Ce rôle peut comporter plusieurs facettes. Il peut amener les intervenantes et intervenants à soutenir le groupe lorsque le projet n'avance pas à la vitesse souhaitée ou que des embûches surviennent :

"Parfois, je dois faire des "peptalks", réencourager les gens lorsqu'ils sont découragés. » (ADC 4)

Alors que, d'autres fois, ils peuvent être appelés à jouer un rôle de soutien psychosocial envers des individus en situation de vulnérabilité.

«Ce n'est pas toujours évident. Parfois on travaille avec des gens qui sont très mal pris, qui ont des problèmes au niveau mental; parce qu'on travaille avec tous les individus, alors des problèmes au niveau mental, des problèmes au niveau psychologique, des problèmes au niveau social, des problèmes de pauvreté qui emmènent tous les autres problèmes; il faut que je sois là pour eux également. " (ADC 3)

\section{Éducateur}

Plusieurs agentes et agents de développement considèrent qu'ils ont aussi comme responsabilité de transmettre de l'information à la population et de l'aider à acquérir de nouvelles compétences et connaissances. Certains entretiennent une conception plus classique de leur rôle d'éducateur :

" Je leur montre à faire des demandes de financement, à entrer en contact avec leur conseiller municipal et lui faire part de leurs besoins. » (ADC 6)

Par ailleurs, d'autres ont une conception de leur rôle d'éducateur qui se rapproche davantage de l'éducation populaire ou de la conscientisation :

"Mon rôle d'avoir une influence, de bien informer sur les enjeux sociaux et économiques qui expliquent les problèmes vécus par la population démunie. » (ADC 9)

\section{Planificateur}

Comme le mentionne Lee (2011), un domaine d'expertise propre à l'agente ou agent de développement est celui du processus, de la gestion d'un projet de développement. 
Les gens de la communauté sont les experts de leur situation, mais ils ont besoin des compétences de l'agente ou agent de développement pour concevoir un plan systématique leur permettant de partir de l'identification d'un besoin vécu dans le milieu à un projet concret visant à y répondre. Les participantes et participants à l'étude ont été nombreux à souligner cette compétence comme étant centrale dans leur rôle et leur mandat face à la communauté.

"On aide les gens dans la communauté à découvrir quels sont leurs besoins. Alors ce n'est pas à nous à leur dire : "Tu devrais avoir ça." Alors on travaille avec les gens dans la communauté pour savoir c'est quoi leurs besoins. On fait une évaluation, on pose des questions : C'est quoi que vous avez besoin? Comment est-ce qu'on peut vous aider? Ensuite, on les aide à travailler ensemble à mettre sur pied des projets qui vont demeurer dans le milieu même sans notre présence. » (ADC 5)

"Mon rôle, c'est de leur donner des outils; de développer leur capacité à gérer un projet; leur apprendre à pêcher au lieu de leur donner du poisson; les mettre en contact avec les bonnes personnes dans la communauté; leur apprendre à gérer une réunion, à faire des demandes de financement, à établir des objectifs, pour qu'ils soient autonomes et puissent gérer leurs projets. » (ADC 1)

\section{Réalité organisationnelle}

Les agentes et agents de développement communautaire sont souvent seuls ou très peu nombreux dans leur organisation. Cette situation ne peut que teinter les rapports qu'ils entretiennent avec leurs supérieurs et leurs collègues. Nous avons interrogé les répondantes et répondants sur leur satisfaction concernant l'encadrement qu'ils reçoivent de leur supérieur et sur la nature et la qualité des relations avec les autres membres du personnel de leur organisation.

En général, les agentes et agents de développement communautaire ont exprimé un niveau de satisfaction élevé concernant les liens qu'ils ont autant avec leur supérieur qu'avec leurs collègues. Certains ont mentionné qu'ils voyaient les contacts avec leur patron non pas comme un rapport de contrôle, mais davantage comme un moment permettant de prendre du recul par rapport à leurs interventions et comme une occasion de valider leur compréhension des besoins du milieu et des priorités de l'organisation. Ils mentionnent, pour la plupart, avoir un niveau d'autonomie très élevé et ils sentent qu'ils 
ont la confiance de leur supérieur. En ce qui a trait aux liens qu'ils ont avec les collègues, bien qu'ils les qualifient de positifs, plusieurs mentionnent également que les collègues ne comprennent pas bien la nature exacte de leurs tâches, ce qui peut en inciter certains à entretenir des préjugés négatifs envers leur rôle dans l'organisation :

"Mes collègues ne comprennent pas pourquoi on est toujours absent du bureau. [...] Ils se disent : "Il n'est jamais là... Il n'est jamais là. On ne sait même pas s'il travaille ou pas.” Parce qu'ils ne savent pas pourquoi on est dans la communauté. » (ADC 1)

"Ben, ç'a pris du temps pour que les gens comprennent parce que souvent t`es pas à ton bureau. "Eille, maudit, est-ce qu'il travaille? Est-ce qu'il n'est pas chez lui en train de dormir?” Parce qu'on est souvent en dehors du bureau pour des rencontres avec d'autres partenaires. » (ADC 3)

"Il y a quelqu'un qui m’avait posé la question : "Do you really work?” [rire] J'ai dit : "Do you want to know really what I do? Come and go with me." " (ADC 2)

Certains intervenants et intervenantes ont cependant développé des stratégies pour mieux faire connaître leur travail et la réalité de leurs fonctions. Pour cet intervenant, une bonne façon de faire comprendre leurs tâches aux collègues, c'est de susciter leur collaboration dans le cadre d'un projet en particulier, par exemple en faisant du porteà-porte avec eux :

"Là quand ils rentrent, ils disent : "Ouff, c'est fatigant. » Eh ben voilà, c'est ça que je fais moi à chaque fois [...] Là ils comprennent, mais c'n'est pas facile pour certains collègues quand vous leur expliquez pas; ils pensent que vous êtes en train de perdre du temps, vous êtes en train de vous promener dans la communauté et vous ne faites rien. " (ADC 6)

Une autre façon de faire comprendre l'ampleur de leurs tâches, c'est de faire une présentation devant les collègues, lors d'une rencontre d'équipe, sur les différents projets sur lesquels ils travaillent :

"Après que j'ai fait ma liste et que j’ai expliqué ce que je faisais sur tous ces projets, plusieurs m'ont dit qu'ils étaient très impressionnés et qu'ils comprenaient mieux mon travail maintenant. » (ADC 5) 
Lorsque les projets sur lesquels ils travaillent suscitent l'intérêt des médias et font l'objet d'une certaine couverture, cela aide également à faire saisir par les collègues l'importance de leur travail :

"Quand tu vois que les médias s'intéressent beaucoup à ce qu'on fait, puis viennent questionner comment on travaille, la façon dont on travaille aussi, ça impressionne les collègues, et les partenaires aussi. Ça aide à faire comprendre ce que l'on fait et notre importance. » (ADC 3)

\section{Le développement communautaire avec les communautés francophones minoritaires. Une lecture à partir du cadre du capital social}

Travailler avec la population francophone de l'Ontario français colore la pratique de l'intervention communautaire en raison des besoins et des défis spécifiques de cette

"Travailler avec la population francophone de l'Ontario français colore la pratique de l'intervention communautaire en raison des besoins et des défis spécifiques de cette communauté et des organismes ayant pour mission de répondre à ses besoins. " communauté et des organismes ayant pour mission de répondre à ses besoins. Nous aborderons donc, dans cette section, les principaux défis de la communauté francophone du territoire de Champlain, tout en sollicitant les apports théoriques de l'approche du capital social pour dégager les enjeux de l'intervention communautaire avec ce milieu particulier.

\section{Les obstacles au capital social}

Les entrevues avec les intervenantes et intervenants communautaires nous ont permis d'identifier, à l'égard de la réalité et des dynamiques des communautés francophones minoritaires, un certain nombre d'éléments qui constituent des freins et des obstacles au développement et au maintien du capital social chez les communautés francophones minoritaires de la région de Champlain. Le premier élément soulevé est lié à la grande hétérogénéité de la communauté francophone. Cette dernière a beaucoup changé dans les dernières décennies. Auparavant, cette communauté était surtout constituée de francophones de langue maternelle, issus de la migration venue surtout du Québec ou du Nouveau-Brunswick il y a plus d'un siècle. Par contre, aujourd'hui, la communauté francophone est très diversifiée. Les francophones de souche sont toujours nombreux, mais la croissance démographique de la communauté résulte surtout de l'immigration internationale, avec des ressortissants provenant de l'Afrique du 
Nord et de l'Ouest. Ces citoyens ont presque toujours une autre langue que le français comme langue maternelle, et la langue de Molière est souvent la langue officielle qu'ils maîtrisent le mieux. Les enjeux et les défis auxquels font face les différents sous-groupes qui composent la communauté francophone, bien que certains soient communs, peuvent également être différents. Pour les francophones qui vivent en milieu urbain depuis plusieurs générations, qui travaillent et consomment dans des milieux majoritairement anglophones et qui sont souvent aussi à l'aise (et même parfois plus à l'aise) de s'exprimer en anglais qu'en français, la priorité est davantage de recevoir un service répondant à leur exigence et à leurs besoins que de l'obtenir en français. S'ils peuvent recevoir un service (de garde, d'éducation, de loisir, etc.) près de leur domicile et à un coût respectant leur budget, ils vont le prendre, peu importe dans quelle langue officielle il est offert. Une intervenante indique que cette population monte de moins en moins aux barricades pour défendre le droit des francophones à recevoir des services en français. Ainsi, elle affirme :

«Si des services en anglais de qualité supérieure sont disponibles, ils n’hésitent pas à les utiliser. » (ADC 9)

Il y a bien sûr toujours des francophones qui vont défendre les droits des francophones minoritaires. Il existe un regroupement de francophones en Ontario, l'Assemblée de la francophonie de l'Ontario, qui a comme mandat de valoriser et de défendre les intérêts de cette communauté. Toutefois, selon certains répondants et répondantes, sur le terrain, au quotidien, il y a de plus en plus de francophones qui ne s'adressent pas aux

"Il risque de devenir de plus en plus difficile de réclamer que les gouvernements et administrations publiques fassent de l'offre de services en français si les francophones eux-mêmes cessent d'en faire la demande." institutions communautaires, municipales ou provinciales en français, qui ne mentionnent pas vouloir obtenir leurs services en français et qui vont même préférer recevoir leurs services et la documentation en anglais. Il risque de devenir de plus en plus difficile de réclamer que les gouvernements et administrations publiques fassent de l'offre de services en français si les francophones eux-mêmes cessent d'en faire la demande.

Les plus vulnérables dans ce contexte sont les nouveaux arrivants et arrivantes qui ne s'expriment pas en anglais. Ceux qui ne s'expriment ni en anglais ni en français sont dans une situation encore plus précaire. Cependant, le fait de ne pas parler la langue de la majorité, malgré une capacité de communiquer en français, demeure handicapant lorsque, comme nous l'avons vu précédemment, les gens veulent 
se trouver un emploi ou accéder à plusieurs autres services. Selon certains intervenants et intervenantes, les nouveaux arrivants et arrivantes sont souvent très mal à l'aise à l'idée de réclamer des services en français. La position de revendication n'est effectivement pas une position naturelle lorsque des personnes arrivent dans un nouveau pays, que ce soit comme immigrantes ou comme réfugiées. Cette incapacité de s'exprimer en anglais complique énormément leurs démarches d'insertion professionnelle et sociale dans leur nouveau milieu de vie. Dans ce contexte, la préoccupation des personnes est beaucoup plus d'apprendre la langue de la majorité que de défendre le droit de recevoir des services en français. Surtout que leur attachement à la langue française n'est souvent pas le même que celui des personnes qui ont grandi dans un contexte et dans un environnement où la lutte pour les droits linguistiques est valorisée.

"De plus en plus, la francophonie est composée de communautés qui proviennent de l'extérieur du Canada, qui sont de nouveaux arrivants, qui ne s'identifient pas en premier lieu comme francophones, bien souvent, mais peut-être plus comme de nouveaux arrivants. Leurs besoins pourraient être différents de ceux des francophones de souche, ce qui peut même créer des tensions entre les deux communautés. » (ADC 9)

Trouver du travail et accéder à des revenus permettant de répondre aux besoins financiers de la famille est bien plus prioritaire que de défendre les droits des francophones.

"Lorsque chapeautés par une organisation formelle bien établie et accompagnés par des intervenantes et intervenants qui connaissent bien les règles institutionnelles et les acteurs clés à mobiliser et à interpeller, les nouveaux arrivants et arrivantes vont se sentir moins intimidés et plus enclins à faire des revendications."
Il existe cependant des ressources communautaires ayant pour mandat de répondre aux multiples besoins des nouveaux arrivants et arrivantes francophones de la région. Les ADC travaillent souvent en collaboration avec ces ressources pour développer de nouveaux services et favoriser la création de liens entre les membres qui pourraient, autrement, se trouver très isolés. Lorsque chapeautés par une organisation formelle bien établie et accompagnés par des intervenantes et intervenants qui connaissent bien les règles institutionnelles et les acteurs clés à mobiliser et à interpeller, les nouveaux arrivants et arrivantes vont se sentir moins intimidés et plus enclins à faire des revendications. Les intervenantes et intervenants communautaires peuvent donc jouer un rôle important dans la création et l'entretien de ce genre de liens. Comme le mentionne un répondant : 
"Seul, on se sent faible, mais, en groupe, on se sent beaucoup plus fort. " (ADC 3)

Les personnes aînées francophones constituent, selon les répondantes et répondants, une autre clientèle vulnérable. D'après une intervenante interrogée, ces personnes sont celles qui ont le plus de difficulté à composer avec la situation de la majorité anglophone, car plusieurs d'entre elles ne peuvent pas communiquer en anglais. Certaines étaient parfaitement bilingues lorsqu'elles étaient plus jeunes, mais ont perdu cette capacité après avoir été confrontées à des maladies liées au vieillissement. Une des raisons pour lesquelles les personnes âgées francophones auraient de la difficulté à recevoir des services dans leur langue serait le fait que, selon la répondante, plusieurs services sont pris en charge par le secteur privé, surtout dans le domaine de l'hébergement et des services à domicile :

"De mettre la question de l'accès aux services en français, les droits d'avoir les services en français, c'est vide, parce que ça, c'est un grand marché privé. La plupart des services sont livrés par des organismes privés à qui on peut difficilement imposer des règles comme le bilinguisme. » (ADC 9)

"... diversité importante et récente de la communauté francophone minoritaire de la région de Champlain est en même temps une source de défi et d'opportunité dans une optique de consolidation du capital social."

Nous retenons donc que la diversité importante et récente de la communauté francophone minoritaire de la région de Champlain est en même temps une source de défi et d'opportunité dans une optique de consolidation du capital social. Elle est une source de défi, car les différents sous-groupes qui la composent ont des réalités et des besoins si différents que la création d'un sentiment d'appartenance commun, d'une confiance et de réflexes de réciprocité, nécessaires à la création du bonding entre les membres, prélude à un capital social contributif au développement d'une communauté vulnérable, est difficile à implanter ou à soutenir.

La diversité est cependant également une source d'opportunité, car elle assure une croissance de la francophonie qui ne serait pas envisageable si elle reposait uniquement sur les mêmes sources que dans les décennies précédentes. La " revanche des berceaux " ${ }^{1}$ est un phénomène qui appartient définitivement au passé, et la survie et la dynamisation de la francophonie ontarienne passent maintenant par l'apport de l'immigration. Immanquablement, ces changements dans les caractéristiques identitaires de la 
francophonie devraient inciter les organismes desservant les francophones à adapter leurs mandats et leur offre de services, mais, selon un agent de développement communautaire :

«Certains organismes ne désirent pas modifier leurs services pour accommoder la population changeante, et ces organismes risquent de voir leur pertinence communautaire s'amenuiser rapidement. » (ADC 4)

\section{Les impacts sur la pratique}

Le fait de travailler auprès d'une communauté francophone a un impact sur la pratique de l'intervention en développement communautaire. L'impact se fait sentir de trois façons : le sentiment d'appartenance, le financement des initiatives et la mobilisation des citoyennes et citoyens.

Nous avons déjà constaté que les intervenants et intervenantes francophones ou bilingues développent, très souvent, un sentiment d'appartenance et de responsabilité envers la communauté francophone (de Moissac, et collab., 2015; Savard, et collab., 2013). Les intervenantes et intervenants communautaires se sentent particulièrement interpellés lorsqu'un dossier sur lequel ils travaillent concerne la communauté francophone, car ils ont l'impression de faire partie de cette communauté. Il n'est pas toujours facile non plus de communiquer en anglais des réalités et des vécus qui se vivent en français :

«Moi, j'aimerais transmettre mes émotions en français, ce sont des émotions qui viennent de la communauté. Quand une personne de la communauté en a marre, j'aimerais dire ça qu'il en a marre; j'aimerais transmettre ça, mais quand je le dis en anglais, ça sera différent. Donc, en traduisant avec la ville quand c'est un problème de francophone, il y a toujours une certaine barrière [...] culturelle, linguistique, une certaine barrière d'interprétation due au fait qu'il n'y a pas beaucoup de francophones sur ces instances. » (ADC 1)

Lorsque l'on fait du travail en intervention communautaire, on doit pouvoir accompagner les communautés dans la recherche de solutions à leurs besoins. Une partie de ce travail consiste à apprendre aux regroupements de citoyennes et citoyens à travailler à partir de l'information et des documents produits par les agences gouvernementales ou les fondations privées. Le problème est que ces organisations ne produisent pas toujours leur documentation en français et, lorsqu' elles le font, soit que c'est beaucoup plus tard, soit que le résultat final ne correspond pas aux réalités particulières des communautés francophones. Des intervenantes et intervenants témoignent : 
«On demande des références ou des outils de promotion, et ils nous envoient des pamphlets ou des documents en anglais. Je fais quoi, moi, pour informer mes francophones avec ça? " (ADC 5)

" Je leur dis toujours qu'il faudrait développer une passerelle francophone qui est indépendante de vos produits parce que notre problématique est différente. [...] C'est parce que l'outil que vous allez traduire ne correspond pas à, probablement à notre réalité sur le terrain. » (ADC 2)

Les intervenantes et intervenants communautaires rencontrés expliquent également qu'il n'est pas toujours facile de faire reconnaître l'importance des besoins vécus par les communautés francophones. Les instances qui financent les projets reconnaissent que les francophones vivent des réalités particulières, mais ils ne reconnaissent pas nécessairement que ces besoins doivent être considérés prioritairement :

«Ils comprennent qu'il y a vraiment des besoins francophones, mais [...] ce n'est [pas] visible. Vous voyez qu' ils veulent nous couper tout ce qu' il y a comme projet [...], mais quand vous coupez ça, ça fait que vous avez compris qu'il y a un besoin, mais vous n'acceptez pas que ce besoin-là soit nécessaire. » (ADC 1)

Donc, être une agente ou un agent de développement communautaire qui travaille avec les communautés francophones implique la nécessité de constamment militer pour le droit des francophones à recevoir du financement pour les projets qui les concernent, même si le nombre de personnes touchées ne semble pas justifier le financement. Car le problème, il est souvent là, pour les organismes subventionnaires; le nombre de personnes touchées est souvent plus important que la langue qu'elles parlent. Une répondante résume ainsi la situation :

«Lorsque le développement est une vision du marché où l'on cherche toujours à répondre à une logique du plus grand nombre (système privé) — parce que c'est quelque part ça qui est la priorité — , il est très difficile de mettre la priorité sur les francophones, compte tenu qu'ils sont $20 \%$ de la population et qu'ils sont répartis à la grandeur du territoire. » (ADC 9)

La langue s'avère également un obstacle à la création de liens entre les agentes et agents de développement communautaire qui travaillent dans les différents centres de ressources communautaires de la ville. La majorité des intervenantes et intervenants sont anglophones, et les francophones doivent souvent accepter de faire les rencontres 
en anglais, au détriment de leur capacité à exprimer, avec toutes les nuances voulues, leur point de vue. Cependant, pour assurer une visibilité au français à l'intérieur de leur réseau, les agentes et agents francophones ont développé certaines stratégies :

"Je fais l'effort de faire savoir que je parle français, que je suis francophone. Donc, dans des meetings [...], c'est de valeur, mais souvent ça se passe en anglais. [...] Quand ça se passe, je dis : "Si jamais vous avez besoin de traduction ou vous voulez le dire en français, moi ça va me faire plaisir de le répéter en anglais pour que les autres comprennent. Sentez-vous bien à l'aise." Parfois, on va lancer des commentaires en français, juste pour dire que c'est correct de parler français là. » (ADC 3)

Ces stratégies ont certainement leur pertinence pour assurer une place au français, mais elles peuvent nuire à la création du lien entre les agentes et agents de développement communautaire de la région, car la langue devient un élément qui divise et qui amenuise le sentiment de solidarité entre les intervenantes et intervenants. Le capital social des agentes et agents de développement communautaire est donc négativement affecté par cette scission linguistique. Par contre, les rencontres du réseau peuvent s'avérer un lieu important pour sensibiliser d'autres actrices et acteurs aux enjeux et aux réalités qui touchent les populations francophones et donc favoriser le bridging entre la communauté francophone et les autres communautés de la région.

\section{La mobilisation et la participation citoyenne}

Le succès d'un projet de développement communautaire repose souvent sur la capacité de mobiliser les citoyennes et citoyens concernés par le besoin ou le problème ciblé. Les théoriciens de l'intervention collective s'entendent généralement pour dire que les bienfaits d'un projet communautaire sont beaucoup plus importants lorsque la communauté ciblée s'implique dès le début du projet et à toutes les étapes ultérieures. Cependant, il n'est pas facile de mobiliser la communauté francophone, car, comme nous l'avons indiqué plus tôt, elle est confrontée à des défis qui sont loin de favoriser un tel engagement. La grande diversité des caractéristiques de cette collectivité est certainement un obstacle à souligner. Comme le mentionnait Saul Alinsky dans son célèbre Reveille for Radicals, une personne sera encline à s'engager dans une initiative communautaire si elle se sent concernée par le problème ou le besoin visé par la démarche. Mais quel enjeu saura mobiliser l'ensemble de la communauté, étant donné que celle-ci est composée de nombreux sous-groupes aux besoins très disparates? 
La grande dispersion géographique des francophones est un autre obstacle important à la mobilisation. Traditionnellement, la communauté francophone était concentrée à l'est et dans le centre-ville d'Ottawa. Bien que l'on retrouve encore dans ces secteurs les plus grandes concentrations de francophones, on retrouve aujourd'hui des contingents importants de cette communauté linguistique dans plusieurs autres parties de la ville. Leur dispersion sur l'ensemble du territoire fait en sorte qu'ils ne semblent plus aussi visibles qu'auparavant. Un agent de développement communautaire s'exprime ainsi sur cet enjeu :

"Où sont les francophones? Ils ne sont pas dans les quartiers à faible revenu de mon secteur et ils ne viennent pas pour les services. Alors, "Where are they?" " (ADC 5)

"En plus du manque de visibilité, l'absence de concentration géographique rend beaucoup plus difficile le rassemblement des populations autour d'une action. Les gens doivent se déplacer, ce qui nécessite temps et ressources financières, deux éléments qui font cruellement défaut parmi les groupes vulnérables, francophones ou non."
En plus du manque de visibilité, l'absence de concentration géographique rend beaucoup plus difficile le rassemblement des populations autour d'une action. Les gens doivent se déplacer, ce qui nécessite temps et ressources financières, deux éléments qui font cruellement défaut parmi les groupes vulnérables, francophones ou non. De plus, si la communauté francophone identifie la nécessité d'obtenir une nouvelle ressource pour répondre à ses besoins, où va-t-on la localiser si on parvient à trouver le financement pour la créer? Dans l'est, où les francophones sont de moins en moins nombreux, mais tout de même plus concentrés, ou, dans l'ouest, où ils s'installent de plus en plus, mais sont toujours encore très faiblement densifiés?

\section{Discussion et conclusion}

Nous avons pu montrer, dans les sections précédentes, que la pratique du développement des communautés en Ontario francophone comporte plusieurs défis. Tout d'abord, la profession doit composer avec des pourtours mal définis. Il n'y a pas de formation académique ayant pour but premier de préparer les futurs intervenants et intervenantes à assumer leurs fonctions, comme c'est le cas pour les infirmières et infirmiers, et les travailleuses et travailleurs sociaux, par exemple. La transmission d'un bagage collectif de valeurs, de principes, de compétences et de meilleures pratiques est donc rendue 
beaucoup plus difficile. Cela explique probablement en partie pourquoi, à l'intérieur même de leur organisation, beaucoup de leurs collègues ont une idée très vague de leurs rôles et de leurs fonctions. Cette réalité rend beaucoup plus vulnérable leur situation dans les organisations qui les emploient. Qui, en effet, va défendre une profession et une pratique mal définies et mal connues? Pourtant, lorsque l'on écoute les agentes et agents de développement parler de leur travail avec les communautés francophones minoritaires, de leur rôle et de leur mission auprès de cette population vulnérable, on constate qu'ils ont un rôle très important à jouer et qu'ils peuvent contribuer de façon considérable au développement et à l'enrichissement de cette communauté.

En guise de discussion et de conclusion de cet article, nous allons nous inspirer des travaux de Ruuskanen (2001), ainsi que du discours des intervenantes et intervenants rencontrés dans le cadre de l'étude dont nous venons de présenter les principaux résultats, pour dégager les conditions qui s'avèrent des atouts et des limites au développement et au maintien du capital social des communautés francophones minoritaires qui évoluent

"...la capacité d'une communauté minoritaire... à survivre et à prospérer, que ce soit au niveau économique, social ou politique, n'est possible que si elle bénéficie d'un capital social suffisant pour soutenir sa capacité à relever les défis collectifs auxquels elle est confrontée." dans la grande région de Champlain, en Ontario. Cette réflexion se justifie par la prise en considération d'un postulat central dans notre questionnement, à savoir que la capacité d'une communauté minoritaire - donc, par définition, vulnérable, sauf en de rares exceptions - à survivre et à prospérer, que ce soit au niveau économique, social ou politique, n'est possible que si elle bénéficie d'un capital social suffisant pour soutenir sa capacité à relever les défis collectifs auxquels elle est confrontée. Comme il est mentionné plus haut, la contribution la plus importante de Ruuskanen a été de proposer une catégorisation des différentes dimensions qui composent le capital social. Ainsi, selon lui, le capital social, pour être saisi dans sa plénitude, doit être étudié en fonction des sources qui le nourrissent, des mécanismes qui favorisent son déploiement et des retombées qu'il génère. Revenons brièvement sur ces trois dimensions.

Tout d'abord, examinons les sources que Ruuskanen subdivise en trois niveaux, soit les niveaux micro, méso et macro. Le niveau micro, ou individuel, correspond aux valeurs véhiculées par les membres de la communauté qui favorisent la solidarité et la confiance et qui sont partagées par ceux qui considèrent que leurs intérêts individuels sont intimement liés au bien-être collectif. Le niveau méso, ou communautaire, fait référence aux normes 
de réciprocité qui favorisent les relations et les échanges à l'intérieur et entre les réseaux formels et informels présents dans la communauté. Finalement, le niveau macro, ou sociétal, interpelle les lois et les institutions qui facilitent la libre expression des besoins et qui encouragent le dialogue entre les communautés et les institutions qui les gouvernent.

Les intervenantes et intervenants communautaires rencontrés dans le cadre de la recherche, qui, pour la grande majorité, faisaient partie la communauté francophone, nous ont parlé abondamment de leur sentiment d'appartenance à cette communauté, des liens de solidarité qui existent entre les intervenantes et intervenants et la population francophone, et entre les intervenantes et intervenants francophones. Lorsqu'on leur demande quelles sont les valeurs les plus importantes qu'ils défendent dans leur travail, la solidarité est celle qui est mentionnée le plus souvent. Plusieurs ressources ayant pour mission d'offrir des services aux francophones survivent encore aujourd'hui grâce au bénévolat réalisé par plusieurs de leurs membres, donc grâce au sentiment de solidarité entretenu par ces personnes envers les personnes les plus précaires de leur communauté. La communauté francophone est apte à se mobiliser pour défendre ses intérêts lorsqu'ils sont menacés ou lorsqu'ils ne sont pas suffisamment pris en considération. Cependant, plusieurs éléments menacent cette capacité communautaire à faire front commun. Mentionnons, par exemple, la résistance des organismes à adapter leur mission et leur gouvernance de façon à s'adapter aux changements importants qui traversent la communauté. Les organismes maintiennent des offres de services et des structures de gouvernance laissant croire que la communauté francophone est toujours essentiellement composée de francophones nés au Canada et ayant le français comme langue maternelle. Il suffit d'assister à des assemblées générales annuelles d'organismes communautaires francophones et d'observer la composition des conseils d'administration pour constater que plusieurs organismes tardent à prendre un virage adapté à la nouvelle réalité.

Au niveau macro, plusieurs lois protègent les droits et les intérêts des francophones minoritaires, que ce soit la Loi sur les services en français de l'Ontario, la Charte canadienne des droits et libertés, la Loi sur les langues officielles du Canada, ou la politique du bilinguisme de la Ville d'Ottawa. Ces lois et politiques font en sorte que les francophones sont soutenus dans leur désir d'avoir accès à des services dans leur langue des gouvernements fédéral, provincial et municipal. Elles ont également un fort pouvoir symbolique qui alimente le sentiment de légitimité de la communauté francophone minoritaire. Elles donnent aussi des outils pour soutenir la défense des intérêts des francophones qui considèrent que leurs droits ne sont pas respectés. L'accès à des services en français n'est pas totalement garanti par les lois, mais celles-ci donnent des outils pour faire progresser 
"La qualité de la mobilisation des communautés, ellemême influencée par limportance du capital social qu'elle déploie, va souvent déterminer la capacité de la communauté francophone à obtenir les ressources dont elle a besoin."

l'offre de services en fonction de l'évolution des besoins des francophones. La qualité de la mobilisation des communautés, elle-même influencée par l'importance du capital social qu' elle déploie, va souvent déterminer la capacité de la communauté francophone à obtenir les ressources dont elle a besoin.

La deuxième dimension concerne les mécanismes qui favorisent le développement du capital social. Ces mécanismes, selon Ruuskanen (2001), permettent d'abord de développer la confiance entre les membres (bonding) et entre la communauté et les autres groupes et instances situés à l'extérieur (bridging). Les mécanismes favorisent ensuite la communication ouverte entre les membres, ce qui assure une meilleure connaissance et un accès à de l'information riche et diversifiée sur les enjeux et les opportunités. Il semble exister plusieurs mécanismes dans la région permettant aux actrices et acteurs de la communauté francophone de se réunir et de développer des liens de confiance et une communication ouverte. Le problème est que ces mécanismes ont tendance à regrouper surtout les actrices et acteurs organisationnels et peu les citoyennes et citoyens non affiliés. Cette situation fait que les organisations vont souvent avoir les yeux tournés vers le haut, soit vers les différents ministères et organismes qui les financent, et non vers le bas, c'està-dire vers les besoins de la population qu'elles doivent desservir. Elles sont moins en mesure d'être alertes par rapport aux nouveaux besoins, contraintes et aspirations des francophones, ce qui joue négativement sur les capacités à les rejoindre, à les écouter et à les impliquer dans le développement de leur milieu. Ces facteurs ont, à leur tour, un impact négatif sur le capital social de ces communautés.

Les retombées, toujours d'après le modèle de Ruuskanen, sont nécessairement très différentes. Le réseau des organismes communautaires francophones est de plus en plus intégré (bonding) et branché sur les autres réseaux (bridging). Il est également de plus en plus désincarné, avec tous les risques que cela fait naître pour le capital social des communautés francophones, mais également, selon nous, pour leur survie à long terme. C'est ici que les ADC peuvent jouer un rôle important. Par leurs fonctions, les ADC se doivent d'être en contact direct et constant avec les populations vulnérables de leur territoire. Ce mandat les place donc en excellente position pour constater les changements qui surviennent dans les besoins, les défis et les réalités observables dans ces communautés. Ils sont donc les yeux et les oreilles des centres de ressources communautaires qui les emploient. 


\section{Note}

1 La revanche des berceaux fait référence au phénomène du taux de fécondité très élevé qui a longtemps caractérisé les populations francophones, très majoritairement catholiques, et qui leur a permis de maintenir leur représentativité au Canada, malgré l'absence d'immigration francophone pendant une longue période.

\section{Bibliographie}

BOUCHARD, Louise, et Martin DESMEULES (2011). Minorités de langue officielle du Canada. Égales devant la santé?, Québec, Presses de l'Université du Québec, collection Problèmes sociaux et interventions sociales, $118 \mathrm{p}$.

BOUCHARD, Louise, et collab. (2006). "Capital social, santé et minorités francophones ", Revue canadienne de santé publique, Vol. 97, supplément 2 (mai-juin), p. S17-S21.

BOURDIEU, Pierre (1986). "The forms of capital ", dans J. Richardson (dir.), Handbook of Theory and Research for the Sociology of Education, New York, Greenwood Press, p. 241-258.

BOURQUE, Denis, et René LACHAPELLE (2010). L'organisation communautaire en CSSS, Québec, Presses de l'Université du Québec, 162 p.

BOURQUE, Denis, et collab. (2007). L'organisation communautaire : Fondements, approches et champs de pratique, Québec, Presses de l'Université du Québec, 534 p.

BRIDGE, Simon, Brendan MURTAGH et Ken O'NEILL (2009). Understanding the Social Economy and the Third Sector, Palgrave Macmillan, 306 p.

CARDINAL, Linda, Stéphane LANG et Anik SAUVÉ (2008). "Les minorités francophones hors Québec et la gouvernance des langues officielles : portrait et enjeux ", Francophonies d'Amérique, $\mathrm{N}^{\circ} 26$, p. 209-233.

COLEMAN, James S. (1988). "Social Capital in the Creation of Human Capital », American Journal of Sociology, № 94, p. 95-120.

DE MOISSAC, Danielle, et collab. (2015). Le recrutement et la rétention des professionnels de la santé et des services sociaux bilingues en situation minoritaire, Université de Saint-Boniface-Université d'Ottawa, 56 p.

DROLET, Marie, et collab. (2014). "Health Services for Linguistic Minorities in a Bilingual Setting: Challenges for Bilingual Professionals ", Qualitative Health Research, 24(3), p. 295-305.

DOUCET, Laval, et Louis FAVREAU (1993). Théorie et pratique en organisation communautaire, Québec, Presses de l'Université du Québec, 462 p.

FAVREAU, Louis, et Yves HURTUBISE (1993). CLSC et communautés locales. La contribution de l’organisation communautaire, Québec, Presses de l'Université du Québec, 211 p. 
FORGUES, Éric, Réal ALLARD et Rodrigue LANDRY (2012). «Le partenariat des communautés francophones en situation minoritaire avec l'État ", Minorités linguistiques et société, Vol. 1, p. 180-194.

FORGUES, Éric, Jean-François THIBAULT et Linda CARDINAL (2010). " La gouvernance des communautés francophones en situation minoritaire et le partenariat avec l'État ", Politique et Sociétés, Vol. 29(1), p. 71-90.

GROUPE DE TRAVAIL SUR LES SERVICES DE SANTÉ EN FRANÇAIS (2005). Services de santé pour la communauté franco-ontarienne: feuille de route pour une meilleure accessibilité et une plus grande responsabilité, ministère de la Santé et des Soins de longue durée de l'Ontario, réf. du 9 février 2012, http://www.health.gov.on.ca/fr/common/ministry/publications/reports/fl hs_06/fl hs_06f.pdf

LAMOUREUX, Henri, et collab. (2002). La pratique de l'action communautaire, Québec, Presses de l'Université du Québec, 416 p.

LAMOUREUX, Henri, Robert MAYER et Jean PANET-RAYMOND (1984). L'intervention communautaire, Montréal, Éditions Saint-Martin, 237 p.

LANDRY, Rodrigue, Éric FORGUES et Christophe TRAISNEL (2010). " Autonomie culturelle, gouvernance et communautés francophones en situation minoritaire au Canada ", Politique et Sociétés, Vol. 29, N 1, p. 91-114.

LEE, Bill (2011). Pragmatics of Community Organization, Toronto, CommonAct Press, 305 p.

MARTIN-CARON, Laurence (2013). Recension des écrits sur le capital social et sa mesure, Chaire de recherche Marcelle-Mallet sur la culture philanthropique, Cahier TA1301, 68 p.

OFFICE OF THE COMMISSIONER OF OFFICIAL LANGUAGES (2015). Portrait of Official Languages Groups in the Ottawa Area, Public Works and Government Services, 32 p.

PUTNAM, Robert D. (1995). "Bowling Alone: America’s Declining Social Capital », Journal of Democracy, Vol. 6, № 1 (January), p. 65-78.

RUUSKANEN, Petri (2001). "Trust on the Border of Network Economy ", Social Capital and Trust, Jyväskylä, SoPhi, 245 p.

SANTÉ CANADA (2007). Pour un nouveau leadership en matière d'amélioration des services de santé en français : rapport au ministre fédéral de la Santé, Ottawa, Comité consultatif des communautés francophones en situation minoritaire, $58 \mathrm{p}$.

SAVARD, Sébastien, et collab. (2013). «Les professionnels de la santé et des services sociaux intervenant auprès des francophones minoritaires : l'enjeu du capital social ", Francophonies d'Amérique, No 36, p. 113-134. 